\title{
Culture and genome-based analysis of four soil Clostridium isolates reveal their potential for antimicrobial production
}

Amila S. N. W. Pahalagedara 1,2,3, Ruy Jauregui ${ }^{3,4}$, Paul Maclean ${ }^{3,4}$, Eric Altermann 1,2,4, Steve Flint ${ }^{2,3}$, Jon Palmer ${ }^{2,3}$, Gale Brightwell ${ }^{1,2,5}$ and Tanushree Barua Gupta ${ }^{1,2^{*}}$

\begin{abstract}
Background: Soil bacteria are a major source of specialized metabolites including antimicrobial compounds. Yet, one of the most diverse genera of bacteria ubiquitously present in soil, Clostridium, has been largely overlooked in bioactive compound discovery. As Clostridium spp. thrive in extreme environments with their metabolic mechanisms adapted to the harsh conditions, they are likely to synthesize molecules with unknown structures, properties, and functions. Therefore, their potential to synthesize small molecules with biological activities should be of great interest in the search for novel antimicrobial compounds. The current study focused on investigating the antimicrobial potential of four soil Clostridium isolates, FS01, FS2.2 FS03, and FS04, using a genome-led approach, validated by culture-based methods.

Results: Conditioned/spent media from all four Clostridium isolates showed varying levels of antimicrobial activity against indicator microorganism; all four isolates significantly inhibited the growth of Pseudomonas aeruginosa. FS01, FS2.2, and FS04 were active against Bacillus mycoides and FS03 reduced the growth of Bacillus cereus. Phylogenetic analysis together with DNA-DNA hybridization (dDDH), average nucleotide identity (ANI), and functional genome distribution (FGD) analyses confirmed that FS01, FS2.2, and FS04 belong to the species Paraclostridium bifermentans, Clostridium cadaveris, and Clostridium senegalense respectively, while FS03 may represent a novel species of the genus Clostridium. Bioinformatics analysis using antiSMASH 5.0 predicted the presence of eight biosynthetic gene clusters (BGCs) encoding for the synthesis of ribosomally synthesized posttranslationally modified peptides (RiPPs) and non-ribosomal peptides (NRPs) in four genomes. All predicted BGCs showed no similarity with any known BGCs suggesting novelty of the molecules from those predicted gene clusters. In addition, the analysis of genomes for putative virulence factors revealed the presence of four putative Clostridium toxin related genes in FSO1 and FS2.2 genomes. No genes associated with the main Clostridium toxins were identified in the FSO3 and FSO4 genomes.
\end{abstract}

\footnotetext{
* Correspondence: tanushree.gupta@agresearch.co.nz

'Food System Integrity team, Hopkirk Research Institute, AgResearch Ltd,

Massey University, 4474 Palmerston North, New Zealand

${ }^{2}$ School of Food and Advanced Technology, Massey University, 4442

Palmerston North, New Zealand

Full list of author information is available at the end of the article
}

C C The Author(s). 2021 Open Access This article is licensed under a Creative Commons Attribution 4.0 International License, which permits use, sharing, adaptation, distribution and reproduction in any medium or format, as long as you give appropriate credit to the original author(s) and the source, provide a link to the Creative Commons licence, and indicate if changes were made. The images or other third party material in this article are included in the article's Creative Commons licence, unless indicated otherwise in a credit line to the material. If material is not included in the article's Creative Commons licence and your intended use is not permitted by statutory regulation or exceeds the permitted use, you will need to obtain permission directly from the copyright holder. To view a copy of this licence, visit http://creativecommons.org/licenses/by/4.0/ The Creative Commons Public Domain Dedication waiver (http://creativecommons.org/publicdomain/zero/1.0/) applies to the data made available in this article, unless otherwise stated in a credit line to the data. 
Conclusions: The presence of BGCs encoding for uncharacterized RiPPs and NRPSs in the genomes of antagonistic Clostridium spp. isolated from farm soil indicated their potential to produce novel secondary metabolites. This study serves as a basis for the identification and characterization of potent antimicrobials from these soil Clostridium spp. and expands the current knowledge base, encouraging future research into bioactive compound production in members of the genus Clostridium.

Keywords: Clostridium spp., Antimicrobial, Biosynthesis gene clusters, Genome mining, Ribosomally synthesized post-translationally modified peptides, Non-ribosomal peptides

\section{Background}

Antimicrobials are widely used in medicine, food, and agriculture to kill or inhibit the growth of harmful microorganisms [1-3]. Antibiotics have saved lives of millions by controlling infectious diseases [3]. Food industry uses natural and synthetic antimicrobials to control food spoilage and pathogenic bacteria in various food products. However, there is a demand for novel natural antimicrobials for food preservation due to consumer concerns over the adverse health effects of synthetic antimicrobials [2]. The rapid emergence of antimicrobial resistant bacteria poses a challenge for currently available antimicrobial agents used for human and animal infections, and food preservation. The consumer demand and threat of bacterial resistance have emphasized the need for the identification of novel antimicrobial compounds, which may have applications in the food industry and/or medicine from natural sources such as bacteria $[4,5]$.

In the past few decades, bacteria have been considered to be the most promising source of antimicrobials [6]. Soil is one of the most complex ecosystems harbouring a large and diversified group of bacteria. Soil microorganisms have developed various strategies to survive and multiply under the variable abiotic and biotic conditions of soil. The production of bioactive molecules such as antimicrobials is a survival mechanism for these microorganisms [7]. Soil actinomycetes have been screened for antimicrobials and they produce more than $80 \%$ of the known antimicrobials used in today's medicine and other applications. However, soil anaerobes such as Clostridium species, which are metabolically diverse and play an important role in natural processes such as degradation of waste, fixation of carbon dioxide, and fermentation of organic matters, have not been thoroughly investigated for their antimicrobial production [8]. This might be due to the long-standing assumption that anaerobes were not capable of producing secondary metabolites such as polyketides and non-ribosomal peptides [9]. Perhaps, the pathogenicity of some members of the genus attracted more attention than the beneficial effects of non-pathogenic Clostridium species. Their metabolic diversity suggests they are of interest in exploring the potential for novel secondary metabolites possessing antimicrobial properties.
In recent years, the development of in silico strategies for the prediction of potential gene clusters, involved in the synthesis of bioactive compounds, has permitted a new understanding of the antimicrobial potential of various microorganisms from different environments. Genome sequencing and rapidly developing bioinformatics tools/methods provide opportunities to assess the biosynthetic potential of microorganisms prior to any biological testing or chemical analysis. Genome mining for the existence of biosynthetic pathways that allow microorganisms to synthesise secondary metabolites including antimicrobials has therefore become part of the emerging efforts to renew antimicrobial discovery. The genes responsible for the synthesis of secondary metabolites in bacteria are often organized as biosynthetic gene clusters (BGCs). These gene clusters usually consist of genes responsible for biosynthesis of precursors, assembly and modification of compound scaffolds, and genes required for regulation, export, and resistance [10]. BGCs are classified based on their products; ribosomally synthesized post -translationally modified peptides (RiPPs), non-ribosomal peptide synthetases (NRPSs), and polyketide synthases (PKSs). NRPSs and PKSs encode for modular enzymes that are involved in the biosynthesis of a large class of structurally diverse, clinically important bioactive compounds including antimicrobials [11].

Several computational tools including antiSMASH [12], ARTS [13], BAGEL 3 [14], Np.searcher [15], RODEO [16], and SMURF [17] have been developed to predict BGCs in bacterial and fungal genome sequences. Among them, antiSMASH (antibiotics and secondary metabolite analysis shell) has become a popular tool used widely to identify secondary metabolite BGCs in the genomes of interest [12]. This could be due to antiSMASH's broad collection of tools and databases for genome mining, BGC analysis and domain analysis for a variety of secondary metabolites, and is a free and public webserver tool [10]. antiSMASH utilizes pHMMs (profile Hidden Markov Models) signature genes and PKS/NRPS signature domains for the detection of putative biosynthetic gene clusters in query genomes. Moreover, antiSMASH generated results are used by several other independent tools including ARTS (Antibiotic Resistance Target Seeker) and BigSCAPE (BGC clustering and classification platform) [18]. 
Our previous study revealed the antimicrobial potential of Farm 4 soil conditioned medium originating from FS01, FS2.2, FS03, and FS04 Clostridium isolates [19]. In the present study, we investigated the under-explored biosynthetic potential of these Clostridium spp. for the synthesis of secondary metabolites belonging to antimicrobial compound groups and provided genome and culture-based evidence for their antimicrobial potential. Two isolates (FS01 and FS03) were sequenced in the current study and the other two isolates (FS2.2 and FS04) were previously sequenced $[20,21]$. Culture dependent work demonstrated the antimicrobial properties of all four soil Clostridium isolates and the genomebased study revealed the presence of putative secondary metabolite gene clusters defining the biosynthetic capability of four soil Clostridium spp. to produce secondary metabolites, with potential antimicrobial properties.

\section{Results and discussion}

Growth inhibitory activity of four soil Clostridium isolates In order to screen Clostridium isolates from soil for antimicrobial activity, conditioned media of all four Clostridium isolates were prepared after growing in cooked meat glucose starch medium (CMGS) as monocultures and tested for growth inhibition of Bacillus mycoides ATCC6462, Bacillus cereus NZRM5, and Pseudomonas aeruginosa ATCC25668. These bacteria were selected based on their adverse impact on food safety and quality, and human health. B. cereus has been reported to cause two types of food poisoning: diarrhoeal and emetic [22, 23]. B. mycoides is mainly associated with food spoilage and their food poisoning potential appears to be low [24]. Therefore, limiting the growth of these bacteria is important to maintain the safety and quality of food products. P. aeruginosa is a known opportunistic pathogen and a major cause of hospital acquired infections. Its control has become challenging due to resistance to multiple antimicrobial drugs [25]. P. aeruginosa has also been identified as a spoilage bacterium in dairy products [26]. Therefore, its control is important in the medical and food industries. FS01CM significantly inhibited the growth of $B$. mycoides and $P$. aeruginosa, but not $B$. cereus in comparison to untreated controls $(P<0.05)$. Similarly, FS2.2CM was active against $B$. mycoides and $P$. aeruginosa. Among all four isolates, the strongest and broadest antimicrobial activity was reported from FS03CM showing significant growth inhibition against all three test microorganisms compared to untreated controls $(P<0.05)$. FS04CM demonstrated the least activity by inhibiting only the growth of $P$. aeruginosa (Fig. 1). The positive control, nisin, showed strong antimicrobial activity against Gram-positive Bacillus spp., but not against Gram-negative $P$. aeruginosa, consistent with previous reports [27, 28]. In summary, conditioned media from all four Clostridium isolates showed various levels of antimicrobial activity against test microorganisms. Interestingly, all four CMs from the four Clostridium isolates produced stronger activity against $P$. aeruginosa than the widely used commercial food preservative nisin. However, as only a single growth condition was used to prepare the conditioned media from Clostridium isolates, this may not demonstrate their full antimicrobial potential. A single laboratory condition may not trigger the production of some specific metabolites in bacteria as their activation may require specific abiotic and/or biotic environmental cues [29].

\section{Genome sequencing and taxonomic characterization}

The genomes of all four soil Clostridium isolates were sequenced using the Illumina MiSeq version 3 sequencing platform. The size of draft assembled genomes of the four Clostridium isolates ranged from 3.5 to $3.9 \mathrm{Mb}$ in line with the size of previously reported clostridial genomes [30], and their respective genomic features are summarized in Table 1. Genome data of FS01, FS2.2, FS03, and FS04 isolates were submitted to NCBI under the accession number PRJNA705805, PRJNA642910, PRJNA706025, and PRJNA605262 respectively.

A preliminary identification of four soil isolates was carried out using PCR based $16 \mathrm{~S}$ rRNA gene sequence analysis as described previously [19]. This indicated that FS01, FS2.2, FS03, and FS04 were taxonomically closely related to Paraclostridium bifermentans (98.1\%), Clostridium cadaveris (100\%), Terrisporobacter glycolicus (95.6\%), and Clostridium senegalense (98.2\%) respectively. In the current study, whole genome sequences of these four soil Clostridium isolates were used to further confirm the taxonomic assignment and the functional relationships between the isolates and closely related microorganisms.

A phylogenetic tree was constructed using extracted the $16 \mathrm{~S}$ rRNA gene sequences from whole genome sequences and indicated that FS01 clustered together with Paraclostridium bifermentans and Paraclostridium benzolyticum; FS2.2 was closely related to Clostridium cadaveris; FS03 was closely related to Terrisporobacter glycolicus, and FS04 was closely related to Clostridium senegalence (Fig. 2). These phylogenetic neighbours were similar to those previously reported by PCR based $16 \mathrm{~S}$ rRNA gene sequence results from all four isolates. $C$. cadaveris genomes were manually added to the TYGS analysis due to the absence of any draft genome sequence of its type strains in the TYGS database.

Genome-based methods including in silico digital DNA-DNA hybridization ( $\mathrm{dDDH})$ and average nucleotide identity (ANI) have been identified as important 

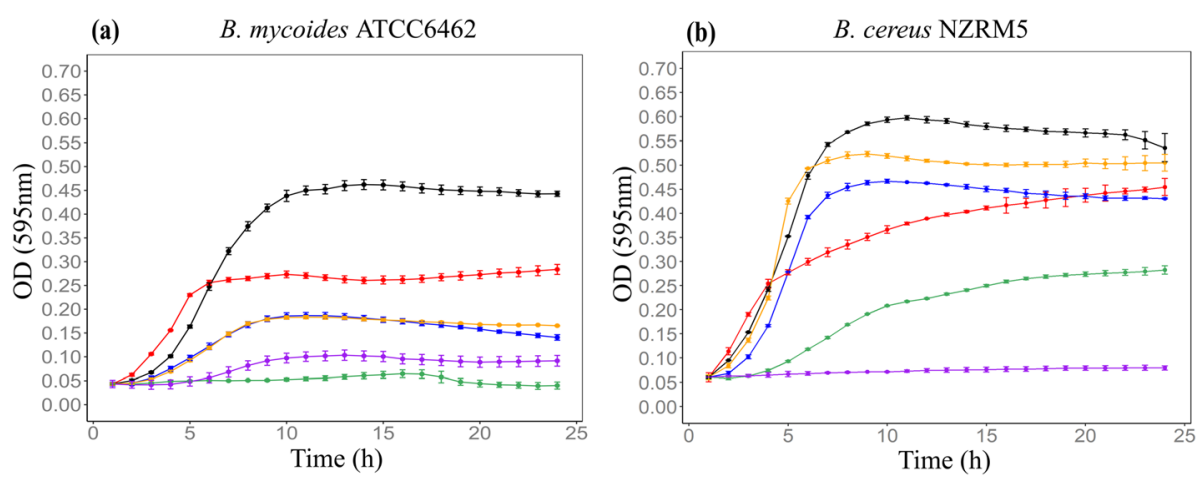

(c) P. aeruginosa ATCC25668

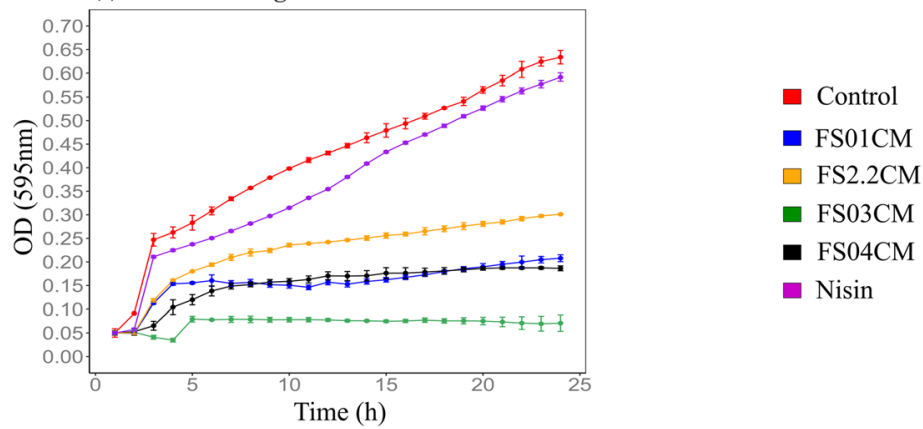

Fig. 1 Effect of conditioned media derived from four soil Clostridium isolates on the growth of B. mycoides ATCC6462 (a), B. cereus NZRM5 (b) and P. aeruginosa ATCC25668 (c). Bacteria were grown in the presence of butterfield's diluent (untreated control; red), FS01CM (FS01 conditioned media; blue), FS2.2CM (FS2.2 conditioned media; orange), FS03CM (FS03 conditioned media; green), FS04CM (FS04 conditioned media; black) and Nisin (purple) in the growth media (CMGS). Nisin $(45 \mu \mathrm{M})$ and butterfield's diluent served as positive and untreated control respectively. Error bars represent standard deviation $(n=3)$. The growth of $P$. aeruginosa was significantly reduced by all four CMs, while only FSO3CM significantly inhibited the growth of $B$. cereus and there was a significant growth reduction of B. mycoides by FS01CM, FS2.2CM and FS03CM $(P<0.05$ vs. untreated control)

criteria in microbial species delineation [31, 32]. These values have been coined as the overall genome related index (OGRI) [33]. Digital DNA:DNA hybridization values for each genome of the Clostridium isolates and their closest neighbours are shown in Table 2. ANI values, which can be used as a measure of genetic relatedness between genomes, were also calculated for the same genome pairs [31]. dDDH values and ANI values obtained for FS01 and Paraclostridium bifermentans (formerly known as Clostridium bifermentans), FS2.2 and Clostridium cadaveris, FS04 and Clostridium senegalense were over the recommended species boundary cut-off values of $70 \% \mathrm{dDDH}$ and $95 \%$ ANI, respectively $[31,34]$. These results indicate that these three isolates

Table 1 General features of the genomes used in this study

\begin{tabular}{lllll}
\hline & Isolate ID & & & FS03 \\
\cline { 2 - 5 } & FS01 & FS2.2 & $3,953,295$ & FS04 \\
\hline Draft Genome size (bp) & $3,529,942$ & $3,620,293$ & 83 & $3,984,260$ \\
Completeness (\%) & 98 & 97 & 28 & 27 \\
G+C (mol\%) & 28 & 31 & 3900 & 38 \\
Genes (total) & 3541 & 3614 & 3752 & 3403 \\
CDSs (total) & 3375 & 3489 & 3719 & 3430 \\
Genes (coding) & 3363 & 3428 & $13,19,18$ & $11,19,16$ \\
rRNAs (5 s, 16 s, 23 s) & $16,25,23$ & $9,16,13$ & 94 & 107 \\
tRNAs & 98 & 83 & 32 & 96 \\
Number of contigs & 44 & 88 & 106,458 & 730,680 \\
Contig N50 & $1,993,941$ & 91,086 \\
\hline
\end{tabular}




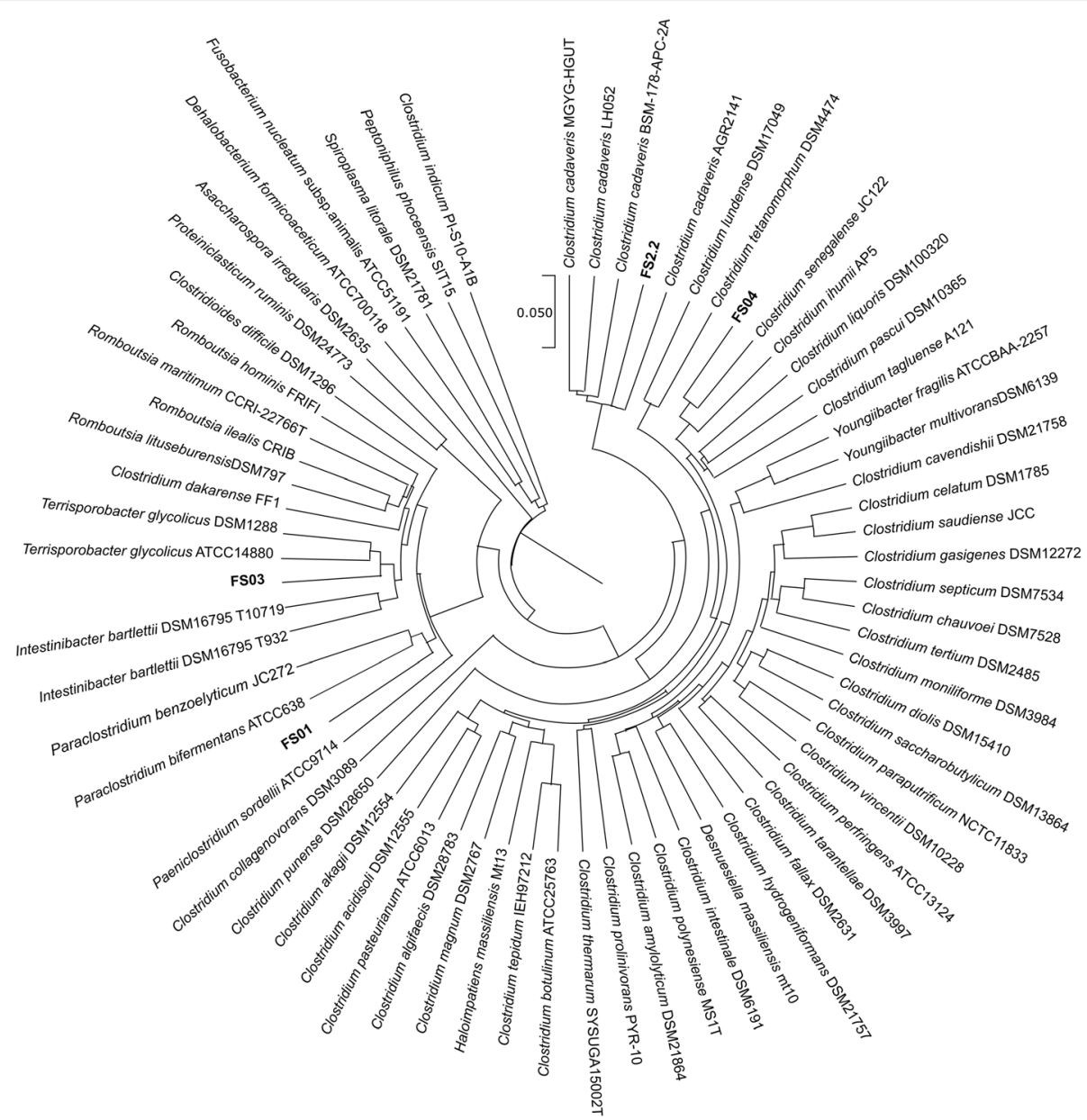

Fig. $216 \mathrm{~S}$ rRNA-based phylogenetic tree inferred with FastME 2.1.6.1 from GBDP distances calculated from $16 \mathrm{~S}$ rRNA gene sequences. The branch lengths are scaled in terms of GBDP distance formula $d_{5}$. Four soil Clostridium isolates are highlighted in bold

belong to the same species they have been compared with (Table 2). dDDH values $(66.8 \%)$ and ANI values $(<$ $95 \%)$ obtained between FS03 and Terrisporobacter glycolicus ATCC 638, and FS03 and Terrisporobacter glycolicus DSM 1288 were below species cut-off values. Therefore, FS03 does not belong to the species T. glycolicus (formerly known as Clostridium glycolicum) even though it is the closest relative according to $16 \mathrm{~S}$ rRNA based phylogenetic analysis. These results indicate that
FS03 may represent a novel species of the genus Terrisporobacter.

A comparative whole genome analysis of four soil isolates and closely related Clostridium spp. was conducted by computing the functional genome distribution (FGD). FGD analysis computes the overall levels of microbial genome similarities by comparing amino acid sequences predicted from each bacterial open reading frames (ORFeomes) [35]. The FGD comparative genomics

Table 2 Intergenomic digital DNA-DNA hybridization and average nucleotide identity values between Clostridium isolates and their closest phylogenetic neighbours

\begin{tabular}{llll}
\hline Isolate & Subject strain & dDDH (CI) in \% & Two-way ANI (\%) \\
\hline FS01 & Paraclostridium bifermentans ATCC638 & $83.2(79.9-86.1)$ & 96.24 \\
FS2.2 & Clostridium cadaveris AGR2141 & $90.2(87-92.7)$ & 99.61 \\
FS03 & Terrisporobacter glycolicus ATCC14880 & $66.8(63.4-70.0)$ & 92.48 \\
& Terrisporobacter glycolicus DSM1288 & $66.8(63.4-70.1)$ & 92.52 \\
FS04 & Clostridium senegalense JC122 & $86.6(83.5-89.2)$ & 95.38 \\
\hline
\end{tabular}


approach carries out genome to genome comparisons highlighting functional associations rather than evolutionary relationships. Based on this analysis, FS01, FS2.2, and FS04 isolates are functionally closely related to their previously assigned species, Paraclostridium bifermentans, Clostridium cadaveris, and Clostridium senegalence respectively (Fig. 3). FS03 showed a close functional relationship to Terrisporobacter glycolicus similar to its TYGS phylogenetic analysis results. However, OGRI suggested that FS03 as a novel species, different from Terrisporobacter glycolicus.

\section{Detection of putative virulence factors (VFs)}

Bacteria exhibiting antagonistic activity against other harmful microorganisms are often considered for developing probiotics. When considering bacteria to be used as probiotic, their pathogenicity is of the utmost important. An array of animal and microbiological assays is available to demonstrate the safety of potential probiotic strains. Nowadays, the evaluation of genome sequences has become a vital part of a thorough safety evaluation [36, 37].
The genus Clostridium includes important human and animal pathogens and some of the members produce exotoxins responsible for diseases such as those causing botulism, tetanus, and gas gangrene. Clostridium perfringens and Clostridium botulinum are two main toxin producers causing foodborne illnesses [38]. In the present study, soil Clostridium isolates shown to possess antimicrobial properties were evaluated for the presence of virulence genes using their whole genome sequences.

The presence of putative virulence genes in all four Clostridium genomes was investigated using VFanalyzer. VFanalyzer is a virulence factor data base (VFDB) integrated automatic pipeline, which can identify known or potential virulence factors in complete or draft genomes of bacteria [39]. The VFanalyser predicted three putative Clostridium toxin related genes; plc (alpha-toxin), colA (kappa-toxin), and $p f o A$ (perfringolysin $\mathrm{O}$ ) in the FS01 genome and one putative toxin related gene; $\operatorname{nagH}(\mathrm{Mu}$ toxin) in the FS2.2 genome. FS03 and FS04 genomes were not found to harbour putative genes for the main Clostridium toxins including alpha-clostripain (cloSI),

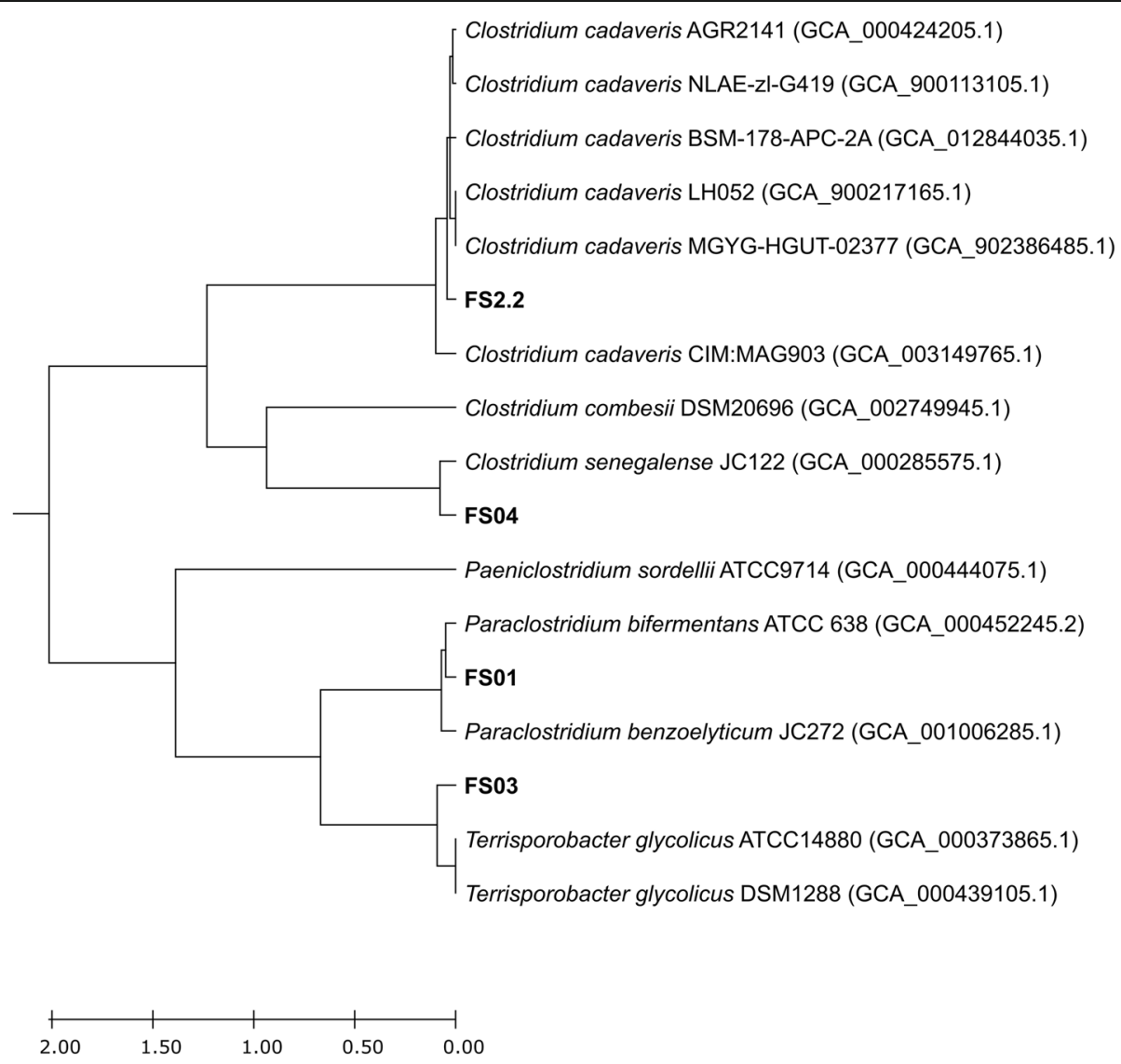

Fig. 3 Inferred phylogenetic tree based on the functional genome distribution (FGD). All the genomes were subjected to FGD analysis, and the resulting distance matrix was imported into MEGA X version 10.2.2. and visualized using unweighted pair group method with arithmetic mean (UPGMA) method 
alpha-toxin ( $p I c)$, beta2 toxin (cpb2), Botulinum neurotoxin (atx), C. novyi alpha-toxin (tcnA), C. perfringens enterotoxin (cpe), Clostridium difficile toxin ( $c d t A, c d t B)$, enterotoxin (ent $A$, entB, entC, entD), kappa-toxin (colA), mu-toxin (nagH, nagI, nagJ, nagK, nagL), perfringolysin $\mathrm{O}(p f o A)$, sialidase (nanH, nanI, nan)), tetanus toxin $($ tet $X)$, toxin $\mathrm{A}(\operatorname{tox} A)$, and toxin $\mathrm{B}(\operatorname{tox} B)$. Based on these results, FS03 and FS04 isolates could be more suitable for developing probiotics as genes for virulence factors were absent. However, the expression of $p l c, \operatorname{col} A$, and $p f o A$ toxin genes detected in FS01 isolate, are usually regulated by the VirS/VirR two component regulatory system [40] and genes responsible for this regulatory system (VirS and VirR) were not found in FS01 genome. However, further assessments are required to confirm their safety to be used as probiotics.

\section{Identification of biosynthetic gene clusters (BGCs)}

In recent years, strict anaerobes such as Clostridium spp. have started to gain attention as potent antimicrobial producers with the advent of computational genomics [29]. Recent studies have focused on obtaining genomic information on the secondary metabolism of anaerobic bacteria including Clostridium species [41-43]. In the current study, four soil Clostridium isolates demonstrated antimicrobial activity against significant food and human associated bacteria and were further investigated for their genetic potential to produce various secondary metabolites, using their assembled whole genome sequences and antiSMASH 5.0 genome mining pipeline.

antiSMASH analysis predicted a total of 8 potential secondary metabolite biosynthetic gene clusters in all four Clostridium genomes (Fig. 4). FS04 showed the highest BGC count detecting one sactipeptide, one
NRPS, and one bacteriocin gene clusters in its genome. FS03 harboured only one BGC, which encoded for a sactipeptide, while the other two genomes (FS01 and FS2.2) featured two BGCs each as shown in Fig. 4. None of the detected clusters could be assigned to a known compound (Table 3). Therefore, it is likely that all of the metabolites synthesised by the detected BGCs have not been fully characterized.

RiPPs gene clusters (sactipeptides and other bacteriocins) The most abundant BGC type annotated in the genomes of the four bacterial isolates was sactipeptide (Fig. 5). Sactipeptides (Sulfur-to-alpha carbon thioether crosslinked peptides) are a class of ribosomally synthesized post-translationally modified peptides (RiPPs). The unique feature of these compounds is the assembling of precursor peptides from encoded genes followed by maturation to the relevant core peptide by introducing at least a one intramolecular thioether bridge, connecting cysteine sulphurs with an unreactive $\alpha$-carbon of the partnering amino acid $[44,45]$. The first reported sactipeptide is subtilosin A from Bacillus subtilis [46]. Since then, more members of this class have been identified from Bacillus species. In recent years, genome mining of various bacterial genomes has revealed the presence of putative sactipeptide biosynthesis gene clusters in other microorganisms including Clostridium spp. [43]. It is postulated that during the maturation process, thioether bond formation is catalysed by a radical $S$-adenosylmethionine (SAM) enzyme encoded by the relevant biosynthetic gene cluster [47]. antiSMASH predicted the core biosynthetic genes of all four sactipeptide clusters using the TIGR03973 HMM profile. Members of TIGR03973 are designated as "Six Cysteines in Forty-

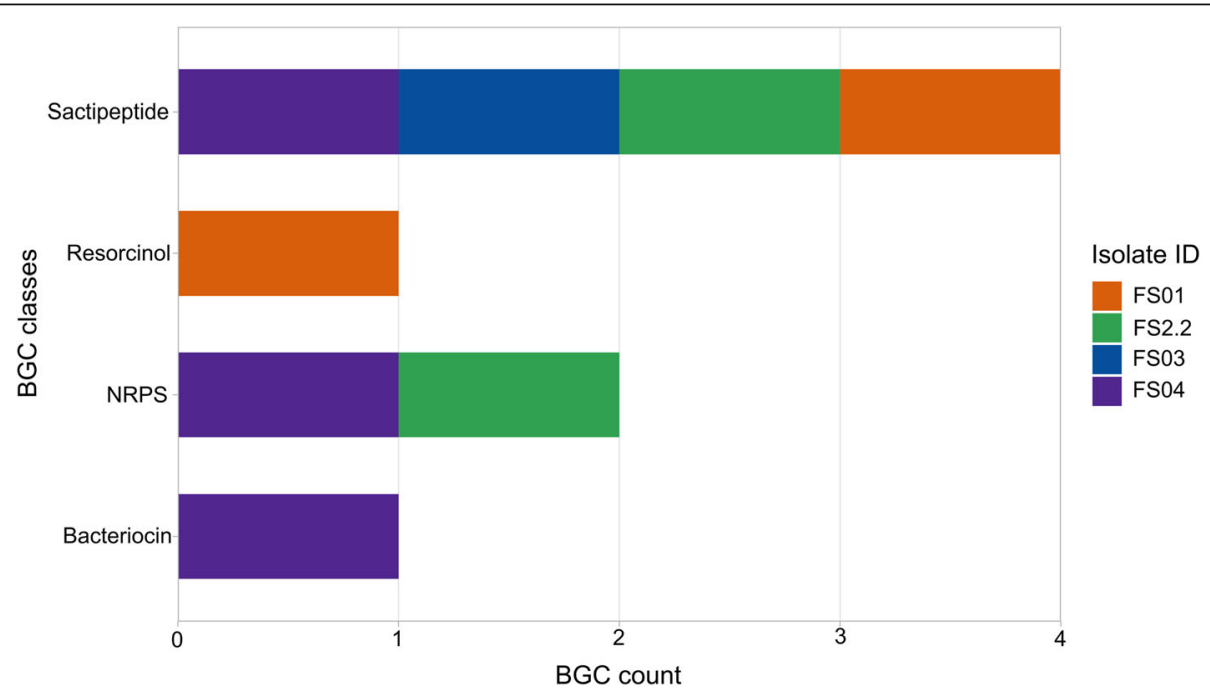

Fig. 4 Total number of different BGCs (BGC count) predicted in all four Clostridium isolates. Different colours depict in which Clostridium isolate they were detected 
Table 3 Details of predicted putative BGCs in all four Clostridium isolates by antiSMASH 5.0

\begin{tabular}{|c|c|c|c|c|}
\hline \multirow[t]{2}{*}{ Isolate } & \multirow[t]{2}{*}{ BGC type } & \multicolumn{2}{|c|}{ Location (nt) } & \multirow{2}{*}{$\begin{array}{l}\text { Most } \\
\text { similar } \\
\text { known } \\
\text { cluster }\end{array}$} \\
\hline & & From & To & \\
\hline \multirow[t]{2}{*}{$\overline{\mathrm{FSO1}}$} & Resorcinol & 157,419 & 198,534 & NA \\
\hline & Sactipeptide & 149,074 & 169,217 & NA \\
\hline \multirow[t]{2}{*}{ FS2.2 } & NRPS & 45,623 & 88,681 & NA \\
\hline & Sactipeptide & 4,427 & 24,633 & NA \\
\hline FS03 & Sactipeptide & 143,920 & 164,063 & NA \\
\hline \multirow[t]{3}{*}{$\mathrm{FSO} 4$} & Sactipeptide & 63,762 & 83,899 & NA \\
\hline & Bacteriocin & 1 & 10,155 & NA \\
\hline & NRPS & 41,090 & 83,957 & NA \\
\hline
\end{tabular}

NA not available

Five residues" (SCIFF) and are predicted ribosomal product precursors linked with an uncharacterized radical SAM protein [48]. The antiSMASH analysis showed that core peptides of predicted sactipeptides were SCIFF belonging to the genus Clostridium and were not associated with any known sactipeptides.

The antiSMASH 'ClusterBlast' tool compares the similarity of individual genes and their arrangement in the query cluster with a comprehensive database of predicted BGCs of publicly available genomes. This comparison identifies other microorganisms harbouring similar BGCs as the query cluster [10]. Furthermore, the 'KnownClusterBlast' module compares any predicted BGCs with the known/ characterized BGCs in the Minimum Information about a Biosynthetic Gene cluster (MIBiG) database to identify the closest compound/ product [49]. The ClusterBlast results indicated that several other Paraclostridium bifermentans strains and Paeniclostridium sordellii strains harbour $100 \%$ identical gene clusters to the predicted FS01 sactipeptide gene cluster in this study (Additional file 1: Fig. S1a). antiSMASH annotated sactipeptide clusters in FS2.2 and FS03 genomes were also found to have $100 \%$ similar gene clusters in Clostridium cadaveris and Terrisporobacter glycolicus strains, respectively. No other bacteria were found to have $100 \%$ identical gene clusters to the
FS04 sactipeptide cluster. The best match was only $94 \%$ gene cluster similarity with Clostridium senegalense. However, only a single gene is potentially different between two gene clusters (Additional file 1: Fig. S1). Since the non-identical gene is located at the margin of the gene cluster and annotated as an 'other' gene, it may not be involved in the sactipeptide biosynthesis. Accordingly, FS04 and $\mathrm{Cl}$. senegalense sactipeptide clusters would be $100 \%$ identical in terms of functionality. Sactipeptide clusters predicted in all four Clostridium genomes showed no similarity to a BGC encoding for a known sactipeptide. This is not surprising as only one sactipeptide cluster, thuricin $\mathrm{CD}$ from Bacillus thuringiensis, has been characterized and included in the MIBiG database so far [49]. To date, no sactipeptide has been chemically identified and isolated from Clostridium spp. [29]. Therefore, these results encourage further studies to identify and characterize sactipeptides from Clostridium species.

Another bacteriocin/unspecified RiPP gene cluster was detected in FS04 (Fig. 6). This gene cluster contains a core gene encoding a protein harbouring a DUF692 domain. Members of the DUF692 protein family are involved in bacteriocin production [50]. An additional biosynthetic gene encoding for a putative peptidase was also annotated in the same gene cluster. There was no other regulatory, transport-related or resistance related gene annotated in the cluster. Cluster blast analysis of the bacteriocin gene cluster did not find a $100 \%$ identical gene cluster in any other bacteria in the antiSMASH database. The bacteriocin cluster showed no relatedness to any known compounds and the most similar cluster was found in the Clostridium senegalense JC122 strain, which showed only $75 \%$ similarity to the FS04 bacteriocin cluster (Additional file 1: Fig. S2).

We cannot further elaborate on the putative functions of these RiPPs as they did not show any relatedness to known compounds. This could be due to the lack of information as only three RiPP clusters from Clostridium spp. have been characterised in the MIBiG database [49]. However, these results expand the knowledge regarding the presence/abundance of potentially novel RiPP gene clusters among species of the genus Clostridium. Further

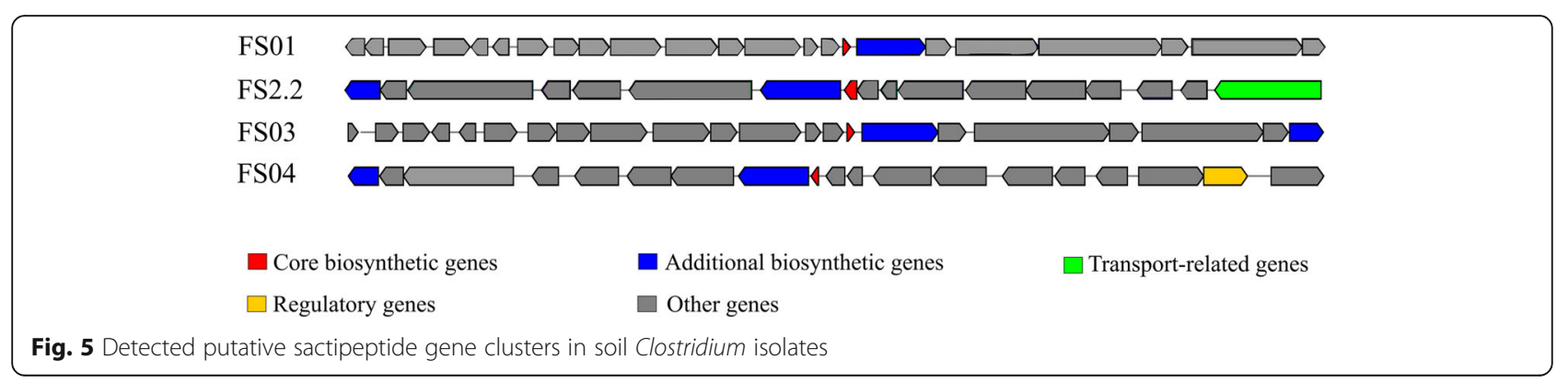




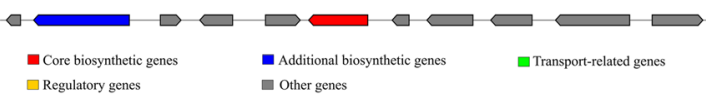

Fig. 6 A bacteriocin gene cluster detected in the FS04 genome

studies are required to understand their products and functions.

\section{NRPS gene clusters}

Microbial products of non-ribosomal peptide (NRP) origin have been significant bioactive compounds possessing antimicrobial, antifungal, anti-tumour properties, and can be used as immunosuppressants [11]. NRPs are synthesized by dedicated non-ribosomal peptide synthetases (NRPSs), which are modular multi-domain enzyme complexes, that serve as templates and biosynthetic machinery for non-ribosomal peptide synthesis [51-53]. NRPSs assemble non-ribosomal peptides through a series of repeating steps that are catalysed by synchronized actions of three catalytic domains: The adenylation domain (A), thiolation or peptidyl carrier protein domain (PCP), and the condensation domain (C) [54]. These catalytic domains when organized together comprise a minimal NRPS module, which is responsible for the incorporation of a single residue into the final peptide product. A fourth domain, a thioesterase, catalyses the release of the peptide from the NRPS [52].
antiSMASH analysis of the four Clostridium isolates in the present study predicted the presence of two putative NRPS gene clusters in the genomes of FS2.2 and FS04. These two gene clusters and their domain organization were found to be different to each other (Fig. 7). Notably, both clusters shared no similarity with those of previously characterized known antimicrobial compounds in the MIBiG database. Furthermore, they appear to be unique NRPS clusters due to the absence of identical clusters in closely related microorganisms. According to cluster blast results, the FS2.2 cluster found the best match with the Clostridium cadaveris AGR2141 genome with $64 \%$ cluster similarity. The NRPS cluster detected in FS04 showed the highest similarity of $55 \%$ to a NRPS cluster in the genome of Clostridium senegalense JC122. These results demonstrate that the predicted clusters may encode producer specific novel NRPS compounds.

In recent years, several studies have predicted the secondary metabolite synthesis potential of anaerobic bacteria including some Clostridium species. A Letzel, SJ Pidot and C Hertweck [43] investigated published genomes of anaerobic bacteria including some Clostridium spp. for the occurrence of RiPP encoding gene clusters and found that over $25 \%$ of the anaerobic genomes tested possessed the genetic basis for producing RiPPs. Another study evaluated 211 published anaerobic genomes in search of specifically putative NRPS and PKS and revealed that $33 \%$ of test genomes possessed

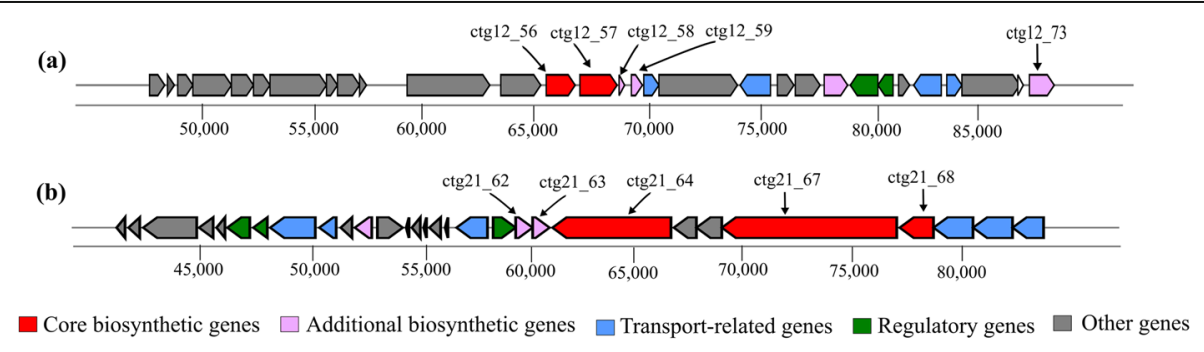

(c)

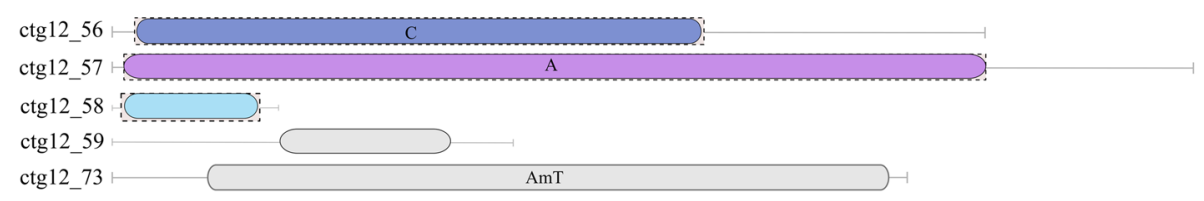

(d)

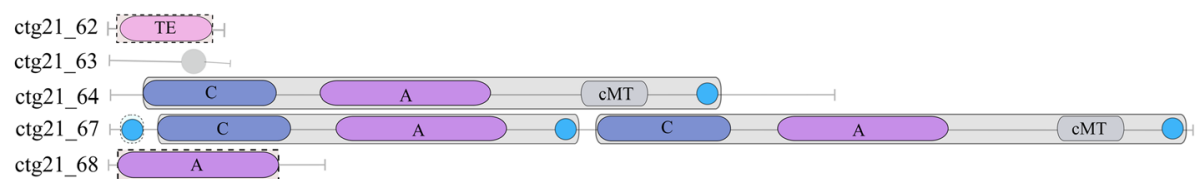

Fig. 7 The predicted NRPS gene clusters. (a) NRPS gene cluster predicted in the FS2.2 genome. (b) NRPS gene cluster detected in the FSO4 genome. (c) Module and domain annotation of the FS2.2 NRPS gene cluster. (d) Module and domain annotation of the FSO4 NRPS gene cluster. Gene locus tags are given on the left side of the domain architecture and in the corresponding genes of the gene cluster 
PKS and NRPS genes [42]. S Behnken and C Hertweck [41] investigated the presence of PKS gene clusters in the genomes of several Clostridium spp. and found a wide distribution of putative PKS gene clusters only in non-pathogenic strains. However, to date, very few antimicrobial secondary metabolites have been identified and characterised from Clostridium species. Therefore, there is limited information on the biosynthetic gene clusters associated with known clostridial antimicrobials limiting the prediction capabilities. In the present study, in agreement with the antagonistic activities, all four Clostridium isolates were predicted to have at least one or more biosynthetic gene clusters in their genomes. All putative gene clusters identified in the present study were not associated with any currently known clostridial or other secondary metabolites in the MiBiG database, indicating that they might encode for novel compounds. Furthermore, some of the putative clusters were unique to the Clostridium isolates used in this study suggesting their respective novelty to produce putative secondary metabolites.

However, it should be taken into consideration that neither the genome-based nor the culture-based approach alone can show the full antimicrobial potential of microorganisms due to their own limitations. Genome mining techniques including antiSMASH are not able to exactly predict the full capacity of a bacterium to produce bioactive compounds, including antimicrobials. Bioinformatic tools such as antiSMASH use BGC prediction algorithms, which are based on the identification of known biosynthetic pathways to some extent and it can be speculated that true novel biosynthetic pathways may not be detected. Most of the genome mining tools use conserved domains of biosynthetic enzymes or pHMMs (profile Hidden Markov Models) signature genes for identification of core genes of a biosynthetic pathway. This implies a limitation of these tools not being able to identify novel pathways and enzymes. To improve the BGC detection, antiSMASH has implemented the 'ClusterFinder' algorithm to identify BGCs that are not detected by the rule-based genome mining. This technique still has some bias towards currently known pathways as BGC detection has been trained using source data from known pathways [10]. The assembly quality of the query genomes is important to achieve reliable results from antiSMASH as its genome analysis relies on identifying genes from the coding regions [10]. On the other hand, the prediction of the presence of a certain biosynthetic pathway does not fully ensure the production of associated secondary metabolites. Biosynthetic gene clusters could be either silent or active under specific growth conditions. Activation of gene clusters may require specific external factors such as presence/ density of other microbes or (lack of) nutrients [55].
However, the limitation in detecting silent metabolic pathways by conventional culture-based methods has been overcome by genome mining. Furthermore, anaerobic bacteria such as Clostridium spp. can utilize a wide range of substrates for their anaerobic fermentation and produce various metabolites based on the substrates used [56]. Some of these small metabolites produced from anaerobic fermentation other than metabolites synthesized as a direct regulation of gene clusters, may also possess antimicrobial activities. For instance, Clostridium difficile was reported to produce paracresol through the fermentation of tyrosine, which showed antagonistic activity against certain bacteria [57]. Despite the above limitations, genome mining has been shown to assist antimicrobial discovery by providing valuable insight into the genomic potential to produce bioactive secondary metabolites.

\section{Conclusions}

BGCs encoding for uncharacterized RiPPs and NRPSs were identified from four soil Clostridium isolates, that showed antimicrobial activities against bacteria associated with human health, food quality and safety, predicting their genetic potential to produce novel secondary metabolites. This knowledge serves as a basis for future investigations to identify and characterize potent antimicrobials from Clostridium isolates and also expands the current knowledgebase on the potential of the genus Clostridium to produce bioactive compounds.

\section{Methods}

\section{Bacterial strains and growth conditions}

All four bacterial isolates (FS01, FS2.2, FS03, and FS04) used in this study were previously isolated from soil collected from bovine dairy farms (Manawatu region in New Zealand) and identified as Clostridium spp. by PCR based $16 \mathrm{~S}$ rRNA gene sequence analysis [19]. Frozen glycerol stocks of these four soil Clostridium isolates were revived on sheep blood agar (SBA) plates following incubation at $35{ }^{\circ} \mathrm{C}$ overnight under anaerobic conditions. Each isolate grown on SBA plates was then used to inoculate either cooked meat glucose starch medium or tryptic soy broth (TSB). Bacillus mycoides ATCC 6462, Bacillus cereus NZRM 5, and Pseudomonas aeruginosa ATCC 25,668 were obtained from Environmental Science and Research (ESR), New Zealand. They were grown in tryptic soy broth (TSB) and incubated at $35{ }^{\circ} \mathrm{C}$ overnight for growth inhibition assays.

\section{Preparation of conditioned medium from soil Clostridium isolates}

Clostridium enriched conditioned media were prepared from all four individual isolates following a previously described method with some modifications [19]. Briefly, 
each Clostridium isolate grown on SBA plates was used to inoculate cooked meat glucose starch medium (45 $\mathrm{mL})$ supplemented with yeast extract $(0.0005 \%)$, hemin $(0.1 \%)$, and vitamin $\mathrm{K}(1 \%)$ and incubated in a $35{ }^{\circ} \mathrm{C}$ anaerobic chamber (Don Whitley Scientific, United Kingdom) for $48 \mathrm{~h}$. After the incubation, enriched media were centrifuged at $10,000 \times \mathrm{g}$ for $40 \mathrm{~min}$ at $4{ }^{\circ} \mathrm{C}$ and supernatants were filter-sterilized using $0.22 \mu \mathrm{m}$ polyvinylidene fluoride syringe filters (Millipore, Ireland). Sterile conditioned media were aliquoted and stored frozen at $-20{ }^{\circ} \mathrm{C}$ until use. Conditioned media prepared from FS01, FS2.2, FS03, and FS04 isolates were denoted as FS01CM, FS2.2CM, FS03CM, and FS04CM respectively.

\section{Growth inhibition assay}

Microplate turbidimetric growth inhibition assays were conducted according to a method described previously [19]. Briefly, an overnight grown bacterial culture was adjusted to $1 \times 10^{7} \mathrm{CFU} / \mathrm{mL}$ cell density and $50 \mu \mathrm{L}$ were mixed with CMGS broth $(50 \mu \mathrm{L})$ and conditioned medium $(100 \mu \mathrm{L})$ in a 96-well flat bottom microtiter plate (Thermo scientific, Denmark) and covered with a sealing membrane (Diversified Biotech, USA). Bacterial growth of the test cultures in each well was assessed by incubating the plate in a microplate spectrometer at 35 ${ }^{\circ} \mathrm{C}$ and measuring the optical density (OD) at $595 \mathrm{~nm}$ for $24 \mathrm{~h}$. Nisin $(45 \mu \mathrm{M})$ (Sigma-Aldrich, USA) and Butterfield's diluent were used as the positive control and untreated control respectively. Corresponding blanks (growth medium, conditioned media, and nisin) were used for background correction.

All experiments were performed in triplicate and data were presented in mean \pm standard deviation. The area under the experimental growth curve (AUC), which represents the overall bacterial growth under given growth conditions was calculated using the $\mathrm{R}$ package 'growthcurver' [58]. Single factor Analysis of Variance (ANOVA) was performed to check the statistical significance of CM treatments on the bacterial growth by comparing the growth in the presence and absence of CM using AUC values. The values with $P<0.05$ were considered statistically significant.

\section{DNA extraction and purification}

Genomic DNA from pure cultures grown in TSB was extracted by using a phenol-chloroform extraction method as described earlier [59] with minor modifications. Bacterial cultures $(5 \mathrm{~mL})$ were centrifuged at $10,000 \times \mathrm{g}$ for $2 \mathrm{~min}$ and the cell pellets were resuspended in $1 \mathrm{~mL}$ TE buffer (composed of $10 \mathrm{mM}$ Tris- $\mathrm{HCl}$ and $1 \mathrm{mM}$ EDTA, $\mathrm{pH}$ 08) (Thermo Fisher Scientific, Lithuania). Resulting cell suspensions were washed twice with TE buffer by centrifuging at 10,000 $\times$ g for $2 \mathrm{~min}$. After washing, harvested cell pellets were resuspended in $200 \mu \mathrm{L}$ genomic DNA solution composed of $1 \mathrm{M}$ sucrose (Fluka, Germany) in TE buffer. Fifty microliters of freshly prepared lysozyme $(50 \mathrm{mg} /$ $\mathrm{mL}$ ) (Sigma-Aldrich, USA) were then added to the cell suspensions and the resulting mixtures were incubated at $37{ }^{\circ} \mathrm{C}$ for $30 \mathrm{~min}$. Following the incubation, $100 \mu \mathrm{L}$ of $20 \%(\mathrm{w} / \mathrm{v})$ sarkosyl (sodium lauroyl sarcosinate) (SigmaAldrich, UK) and $15 \mu \mathrm{L}$ of RNase A (DNase free, $10 \mathrm{mg} /$ $\mathrm{mL}$ ) (Sigma-Aldrich, UK) were added and incubated at $37{ }^{\circ} \mathrm{C}$ for another $30 \mathrm{~min}$. Then, $7.5 \mu \mathrm{L}$ of proteinase $\mathrm{K}$ $(10 \mathrm{mg} / \mathrm{mL})$ (Ambion, USA) were added and incubated at $37^{\circ} \mathrm{C}$ for $30 \mathrm{~min}$. The lysate extraction was carried out three times with $600 \mu \mathrm{L}$ of phenol/chloroform/isoamyl alcohol (25:24:1) ) (Sigma-Aldrich, USA) and centrifuged at $20,000 \times \mathrm{g}$ for $20 \mathrm{~min}$. The DNA was precipitated using $50 \mu \mathrm{L}$ of $3 \mathrm{M}$ sodium acetate ( $\mathrm{pH}$ 5.2) (BDH, UK) and three volumes of ice-cold absolute ethanol at $-20{ }^{\circ} \mathrm{C}$ for overnight, followed by a washing step with $750 \mu \mathrm{L}$ of $70 \%$ ethanol and centrifugation at $17,000 \times \mathrm{g}$ for $5 \mathrm{~min}$. After removing supernatants, genomic DNA pellets were airdried for about $15 \mathrm{~min}$ and re-suspended in $100 \mu \mathrm{L}$ of sterile DNase and RNase free water. All samples were stored at $4{ }^{\circ} \mathrm{C}$ overnight, they were separated by $0.8 \%$ gel electrophoresis, and visualized by Gel Doc XR system (Bio-Rad Laboratories, USA). DNA quality and quantity were assessed using Qubit 4 fluorometer (Thermo Fisher Scientific Inc., USA) and NanoDrop 1000 spectrophotometer (Thermo Fisher Scientific Inc., USA).

\section{Whole genome sequencing, assembly, and annotation}

High quality genomic DNA extracted from four Clostridium isolates was sequenced using Illumina MiSeq version 3 sequencing platform at the Massey University Genome Services, New Zealand. DNA library preparation was performed with the Celero PCR workflow with an enzymatic fragmentation DNA-seq library preparation kit (NuGEN, USA). Quality and quantity assessments of DNA libraries were carried out as a quality control check before sequencing. The resulting reads were quality trimmed, filtered, and de novo assembled with the A5-miseq assembler (version 20,160,825) under the default settings [60]. The genome completeness was assessed using BUSCO (version 1.22) [61].

\section{Phylogenetic and functional genome distribution (FGD) analyses}

A $16 \mathrm{~S}$ rRNA gene sequence based phylogenetic tree was constructed using the Type (Strain) Genome Server (TYGS) webserver [62]. Briefly, draft genomes of FS01, FS2.2, FS03, and FS04 together with four whole genomes of C. cadaveris strains from the NCBI database $\left(\mathrm{GCA}_{-}\right.$ 000424205.1, GCA_900217165.1, GCA 902386485.1 and GCA_012844035.1) were submitted to the TYGS webserver. TYGS extracted available $16 \mathrm{~S}$ rRNA gene sequences 
from query genomes using RNAmmer [63] and then performed NCBI BLAST + analyses using all possible pairs of $16 \mathrm{~S}$ rRNA gene sequences in the TYGS database. The genomes with the top 50 BLAST bitscores were used for the calculation of the Genome BLAST Distance Phylogeny (GBDP) values. GBDP values were calculated between the selected $16 \mathrm{~S}$ rRNA gene sequences under the algorithm 'coverage' and distance formula $d_{5}$ [64], and the closest relatives were defined as the genomes with the lowest ten $16 \mathrm{~S}$ rRNA gene GBDP distances between each query genome and type strains. The resulting GBDP distances were used to construct a $16 \mathrm{~S}$ rRNA gene sequence based phylogenetic tree with FastME 2.1.6.1 including subtree pruning and regrafting (SPR) post processing [65]. The inferred tree was visualized using MEGA X version 10.2.2 [66].

The digital DNA:DNA hybridization $(\mathrm{dDDH})$ values of each of the Clostridium genome and their closest neighbours were computed using Genome-to-Genome Distance Calculator (GGDC version 2.1) in TYGS analysis tool with default parameters [62, 64]. The average nucleotide identity (ANI) values between each Clostridium genome and their closest neighbours were calculated using the ANI calculator developed by Kostas lab with default parameters (http://enve-omics.ce.gatech. edu/ani/). ANI values were calculated as the mean identity of BLASTN matches [31].

Functional genome distribution (FGD) analysis investigates the genomic differences between bacteria and interprets the genetic diversity [35]. FGD analysis was carried out using all four Clostridium isolates and their closest neighbours identified from TYGS phylogenetic analysis. All the genomes required for the analysis except the four Clostridium isolates were downloaded in FASTA format from NCBI database. They were concatenated using a universal spacer-stop-spacer sequence and automatically annotated using GAMOLA 2 software package [67]. FGD analysis was performed using the predicted open reading frames (ORFeomes) of all genomes. The resulting distance value matrix was imported into MEGA X version 10.2.2 and visualized using an unweighted pair group method with arithmetic mean (UPGMA) [66].

\section{Analysis of putative biosynthetic gene clusters (BGCs) and virulence genes}

Biosynthetic gene clusters encoding for secondary metabolites were predicted and annotated using the web server version of antiSMASH 5.0 [12] with default parameters and in combination with the ClusterFinder algorithm, which is a hidden Markov model based probabilistic algorithm to detect BGCs of both known and unknown groups [68]. antiSMASH ClusterBlast and KnownClasterBlast modules were used for comparing detected gene clusters to other sequenced genomes and known gene clusters. The web tool VFanalyzer was used to identify known/potential virulence factors in the genomes of four Clostridium isolates [39].

\section{Abbreviations}

dDDH: DNA-DNA hybridization; ANI: average nucleotide identity; FGD: functional genome distribution; BGCs: biosynthetic gene clusters; RiPPs: ribosomally synthesized post-translationally modified peptides; NRPs: non-ribosomal peptides; NRPSs: non-ribosomal peptide synthetases; PKSs: polyketide synthases; pHMMs: profile hidden markov models; BigSCAPE: BCG clustering and classification platform; PCR: Polymerase chain reaction; TSB: Tryptic soy broth; CMGS: Cooked meat glucose starch medium; OD: Optical density; TYGS: Type (Strain) genome server

\section{Supplementary Information}

The online version contains supplementary material available at https://doi. org/10.1186/s12864-021-08005-2.

\section{Additional file 1.}

\section{Acknowledgements}

We are grateful to AgResearch Ltd. Food System Integrity team at the Hopkirk research institute for their support.

\section{Authors' contributions}

T.B.G and A.S.N.W.P conceived the study. Whole genome sequencing, assembly \& annotation were performed by A.S.N.W.P, RJ, PM. A.S.N.W.P performed genome mining, TYGS phylogenetic analysis and culture-based experiments. FGD analysis was carried out by EA. A.S.N.W.P wrote the first draft and SF, JP, EA, RJ, PM and TBG edited the manuscript. All authors read and approved the manuscript.

\section{Funding}

This work was supported by the Strategic Science Investment Fund (SSIF), AgResearch Ltd., New Zealand.

\section{Availability of data and materials}

Data from the following public databases was used for the analysis.

-Draft genomes from the national (NCBI) assembly database.

https://www.ncbi.nlm.nih.gov/assembly/.

-antiSMASH database.

https://antismash-db.secondarymetabolites.org/.

- Type (Strain) Genome Server (TYGS) database.

https://tygs.dsmz.de/.

Draft whole genome sequences of FS01, FS2.2, FS03 and FS04 isolates were submitted to NCBI under the accession number PRJNA705805, PRJNA642910, PRJNA706025 and PRJNA605262, respectively.

\section{Declarations}

Ethics approval and consent to participate Not applicable.

\section{Consent for publication}

Not applicable.

\section{Competing interests}

The authors declare that they have no competing interests.

\section{Author details}

${ }^{1}$ Food System Integrity team, Hopkirk Research Institute, AgResearch Ltd, Massey University, 4474 Palmerston North, New Zealand. ${ }^{2}$ School of Food and Advanced Technology, Massey University, 4442 Palmerston North, New Zealand. ${ }^{3}$ Data Science team, Grasslands Research Centre, AgResearch Ltd, Palmerston North, New Zealand. ${ }^{4}$ Riddet Institute, Massey University, Palmerston North, New Zealand. ${ }^{5}$ New Zealand Food Safety Science and Research Centre, Massey University, Palmerston North, New Zealand. 
Received: 11 May 2021 Accepted: 13 September 2021 Published online: 22 September 2021

\section{References}

1. Kirchhelle C: Pharming animals: a global history of antibiotics in food production (1935-2017). Palgrave Commun 2018, 4(1):96.

2. El-Saber Batiha G, Hussein DE, Algammal AM, George TT, Jeandet P, Al-Snafi AE, Tiwari A, Pagnossa JP, Lima CM, Thorat ND et al: Application of natural antimicrobials in food preservation: Recent views. Food Control 2021, 126: 108066.

3. Mohr Kl: History of antibiotics research. In: How to Overcome The Antibiotic Crisis: Facts, Challenges, Technologies and Future Perspectives. Edited by Stadler M, Dersch P. Cham: Springer International Publishing; 2016: 237-272.

4. D'Andrea MM, Fraziano M, Thaller MC, Rossolini GM: The urgent need for novel antimicrobial agents and strategies to fight antibiotic resistance. Antibiotics (Basel) 2019, 8(4):254.

5. Santos-Sanchez NF, Salas-Coronado R, Valadez-Blanco R, Hernandez-Carlos B, Guadarrama-Mendoza PC: Natural antioxidant extracts as food preservatives. Acta Sci Pol Technol Aliment 2017, 16(4):361-370.

6. Landwehr W, Wolf C, Wink J: Actinobacteria and myxobacteria-two of the most important bacterial resources for novel antibiotics. In: How to overcome the antibiotic crisis: facts, challenges, technologies and future perspectives. Edited by Stadler M, Dersch P. Cham: Springer International Publishing; 2016: 273-302.

7. Davies J: What are antibiotics? Archaic functions for modern activities. Mol Microbiol 1990, 4(8):1227-1232

8. Schmitz R, Daniel R, Deppenmeier U, Gottschalk G: The anaerobic way of life. In: The Prokaryotes. vol. 2; 2006: 86-101.

9. Behnken S, Hertweck C: Anaerobic bacteria as producers of antibiotics. Appl Microbiol Biotechnol 2012, 96(1):61-67.

10. Blin K, Kim HU, Medema MH, Weber T: Recent development of antiSMASH and other computational approaches to mine secondary metabolite biosynthetic gene clusters. Brief Bioinformatics 2017, 20(4):1103-1113.

11. Walsh CT: The chemical versatility of natural-product assembly lines. Acc Chem Res 2008, 41(1):4-10.

12. Blin K, Shaw S, Steinke K, Villebro R, Ziemert N, Lee SY, Medema MH, Weber T: antiSMASH 5.0: updates to the secondary metabolite genome mining pipeline. Nucleic Acids Research 2019, 47(W1):W81-W87.

13. Alanjary M, Kronmiller B, Adamek M, Blin K, Weber T, Huson D, Philmus B, Ziemert N: The antibiotic resistant target seeker (ARTS), an exploration engine for antibiotic cluster prioritization and novel drug target discovery. Nucleic Acids Research 2017, 45(W1):W42-W48.

14. van Heel AJ, de Jong A, Montalbán-López M, Kok J, Kuipers OP: BAGEL3: automated identification of genes encoding bacteriocins and (non)bactericidal posttranslationally modified peptides. Nucleic Acids Research 2013, 41(W1):W448-W453.

15. Li MHT, Ung PMU, Zajkowski J, Garneau-Tsodikova S, Sherman DH: Automated genome mining for natural products. BMC Bioinform 2009, 10(1):185.

16. Tietz JI, Schwalen CJ, Patel PS, Maxson T, Blair PM, Tai H-C, Zakai UI, Mitchell DA: A new genome-mining tool redefines the lasso peptide biosynthetic landscape. Nat Chem Biol 2017, 13(5):470-478.

17. Khaldi N, Seifuddin FT, Turner G, Haft D, Nierman WC, Wolfe KH, Fedorova ND: SMURF: genomic mapping of fungal secondary metabolite clusters. Fungal Genet Biol 2010, 47(9):736-741.

18. Navarro-Muñoz JC, Selem-Mojica N, Mullowney MW, Kautsar S, Tryon JH, Parkinson El, De Los Santos ELC, Yeong M, Cruz-Morales P, Abubucker S et al: A computational framework for systematic exploration of biosynthetic diversity from large-scale genomic data. bioRxiv 2018:445270.

19. Pahalagedara ASNW, Flint S, Palmer J, Subbaraj A, Brightwell G, Gupta TB: Antimicrobial activity of soil Clostridium enriched conditioned media against Bacillus mycoides, Bacillus cereus, and Pseudomonas aeruginosa. Front Microbiol 2020, 11 (3113).

20. Gupta TB, Jauregui R, Nawarathana AS, Brightwell G, Maclean P: Draft genome sequence of Clostridium senegalense strain AGRFS4, isolated from a dairy farm in New Zealand. Microbiol Resour Announc 2020, 9(19):e00214-00220.

21. Gupta TB, Jauregui R, Maclean P, Nawarathana AS, Brightwell G: Draft genome sequence of Clostridium cadaveris strain AGRFS2.2, isolated from a bovine dairy farm in New Zealand. Microbiol Resour Announc 2020, 9(35): e00787-00720.
22. Prüß BM, Dietrich R, Nibler B, Märtlbauer E, Scherer S: The hemolytic enterotoxin $\mathrm{HBL}$ is broadly distributed among species of the Bacillus cereus group. Appl Environ Microbiol 1999, 65(12):5436-5442.

23. Granum PE, Lund T: Bacillus cereus and its food poisoning toxins. FEMS Microbiol Lett 1997, 157(2):223-228.

24. Bağcıoğlu M, Fricker $M$, Johler S, Ehling-Schulz M: Detection and identification of Bacillus cereus, Bacillus cytotoxicus, Bacillus thuringiensis, Bacillus mycoides and Bacillus weihenstephanensisvia machine learning based FTIR spectroscopy. Front Microbiol 2019, 10:902-902.

25. Bassetti M, Vena A, Croxatto A, Righi E, Guery B: How to managePseudomonas aeruginosainfections. Drugs in Context 2018, 7: 212527.

26. Arslan S, Eyi A, Özdemir F: Spoilage potentials and antimicrobial resistance ofPseudomonasspp. isolated from cheeses. J Dairy Sci 2011, 94(12):5851-5856.

27. Lee N-K, Kim HW, Lee JY, Ahn DU, Kim C-J, Paik H-D: Antimicrobial effect of nisin againstBacillus cereusin beef jerky during storage. Korean J Food Sci Anim Resour 2015, 35(2):272-276.

28. Li Q, Montalban-Lopez M, Kuipers OP: Increasing the antimicrobial activity of nisin-based lantibiotics against Gram-negative pathogens. Appl Environ Microbiol 2018, 84(12):e00052-00018.

29. Pahalagedara ASNW, Flint S, Palmer J, Brightwell G, Gupta TB: Antimicrobial production by strictly anaerobicClostridiumspp. Int J Antimicrob Agents 2020:105910.

30. Brasca M, Morandi S, Silvetti T: Clostridiumspp. In: Reference Module in Food Science. Elsevier; 2020.

31. Goris J, Konstantinidis KT, Klappenbach JA, Coenye T, Vandamme P, Tiedje JM: DNA-DNA hybridization values and their relationship to whole-genome sequence similarities. Int J Syst Evol Microbiol 2007, 57(1):81-91.

32. Varghese NJ, Mukherjee S, Ivanova N, Konstantinidis KT, Mavrommatis K Kyrpides NC, Pati A: Microbial species delineation using whole genome sequences. Nucleic Acids Research 2015, 43(14):6761-6771.

33. Chun J, Rainey FA: Integrating genomics into the taxonomy and systematics of the bacteria and archaea. Int J Syst Evol Microbiol 2014, 64(Pt_2):316-324.

34. Chun J, Oren A, Ventosa A, Christensen H, Arahal DR, da Costa MS, Rooney AP, Yi H, Xu X-W, De Meyer S et al: Proposed minimal standards for the use of genome data for the taxonomy of prokaryotes. Int J Syst Evol Microbiol 2018, 68(1):461-466

35. Altermann E: Tracing lifestyle adaptation in prokaryotic genomes. Front Microbiol 2012, 3(48).

36. Wassenaar TM, Zschüttig A, Beimfohr C, Geske T, Auerbach C, Cook H, Zimmermann K, Gunzer F: Virulence genes in a probiotic E. coli product with a recorded long history of safe use. Eur J Microbiol Immunol (Bp) 2015, 5(1):81-93.

37. Shokryazdan P, Faseleh Jahromi M, Liang JB, Kalavathy R, Sieo CC, Ho YW: Safety assessment of two newLactobacillusstrains as probiotic for human using a rat model. PLoS One 2016, 11(7):e0159851.

38. Clostridium botulinumandClostridium perfringens. In: Foodborne Microbial Pathogens: Mechanisms and Pathogenesis. New York, NY: Springer New York; 2008: 149-164.

39. Liu B, Zheng D, Jin Q, Chen L, Yang J: VFDB 2019: a comparative pathogenomic platform with an interactive web interface. Nucleic Acids Research 2018, 47(D1):D687-D692.

40. Ba-Thein W, Lyristis M, Ohtani K, Nisbet IT, Hayashi H, Rood JI, Shimizu T: The virR/virS locus regulates the transcription of genes encoding extracellular toxin production inClostridium perfringens. J Bacteriol 1996, 178(9):2514-2520.

41. Behnken S, Hertweck C: Cryptic polyketide synthase genes in nonpathogenicClostridiumspp. PLoS One 2012, 7(1):e29609.

42. Letzel A, Pidot SJ, Hertweck C: A genomic approach to the cryptic secondary metabolome of the anaerobic world. Nat Prod Rep 2013, 30(3): 392-428.

43. Letzel A, Pidot SJ, Hertweck C: Genome mining for ribosomally synthesized and post-translationally modified peptides (RiPPs) in anaerobic bacteria. BMC Genom 2014, 15(1):983-983.

44. Flühe L, Marahiel MA: Radical S-adenosylmethionine enzyme catalyzed thioether bond formation in sactipeptide biosynthesis. Curr Opin Chem Biol 2013, 17(4):605-612.

45. Himes PM, Allen SE, Hwang S, Bowers AA: Production of sactipeptides inEscherichia coli: probing the substrate promiscuity of subtilosin A biosynthesis. ACS Chem Biol 2016, 11(6):1737-1744.

46. Kawulka K, Sprules T, McKay RT, Mercier P, Diaper CM, Zuber P, Vederas JC Structure of subtilosin A, an antimicrobial peptide fromBacillus subtiliswith 
unusual posttranslational modifications linking cysteine sulfurs to a-carbons of phenylalanine and threonine. J Am Chem Soc 2003, 125(16):4726-4727.

47. Flühe L, Knappe TA, Gattner MJ, Schäfer A, Burghaus O, Linne U, Marahiel MA: The radical SAM enzyme AlbA catalyzes thioether bond formation in subtilosin A. Nat Chem Biol 2012, 8(4):350-357.

48. Haft DH, Basu MK: Biological systems discoveryIn Silico: radical sadenosylmethionine protein families and their target peptides for posttranslational modification. J Bacteriol 2011, 193(11):2745-2755.

49. Kautsar SA, Blin K, Shaw S, Navarro-Muñoz JC, Terlouw BR, van der Hooft JJJ, van Santen JA, Tracanna V, Suarez Duran HG, Pascal Andreu V et al: MIBiG 2. 0 : a repository for biosynthetic gene clusters of known function. Nucleic Acids Research 2019, 48(D1):D454-D458.

50. Kenney GE, Dassama LMK, Pandelia M-E, Gizzi AS, Martinie RJ, Gao P, DeHart CJ, Schachner LF, Skinner OS, Ro SY et al: The biosynthesis of methanobactin. Science 2018, 359(6382):1411-1416.

51. Lipmann F: Nonribosomal polypeptide synthesis on polyenzyme templates. Acc Chem Res 1973, 6(11):361-367.

52. Felnagle EA, Jackson EE, Chan YA, Podevels AM, Berti AD, McMahon MD, Thomas MG: Nonribosomal peptide synthetases involved in the production of medically relevant natural products. Mol Pharm 2008, 5(2):191-211.

53. Laland SG, Zimmer TL: The protein thiotemplate mechanism of synthesis for the peptide antibiotics produced byBacillus brevis. Essays biochem 1973, 9: $31-57$.

54. Kopp F, Marahiel MA: Macrocyclization strategies in polyketide and nonribosomal peptide biosynthesis. Nat Prod Rep 2007, 24(4):735-749.

55. Gontang EA, Gaudêncio SP, Fenical W, Jensen PR: Sequence-based analysis of secondary-metabolite biosynthesis in marine actinobacteria. Appl Environ Microbiol 2010, 76(8):2487-2499.

56. Huang W-C, Tang IC: Chap. 8 - Bacterial and yeast cultures - process characteristics, products, and applications. In: Bioprocessing for Value-added Products from Renewable Resources. Edited by Yang S-T. Amsterdam: Elsevier; 2007: 185-223.

57. Passmore IJ, Letertre MPM, Preston MD, Bianconi I, Harrison MA, Nasher F, Kaur H, Hong HA, Baines SD, Cutting SM et al: Para-cresol production byClostridium difficileaffects microbial diversity and membrane integrity of Gram-negative bacteria. PLOS Pathogens 2018, 14(9):e1007191.

58. Sprouffske $K$, Wagner A: Growthcurver: an R package for obtaining interpretable metrics from microbial growth curves. BMC Bioinform 2016, 17(1):172.

59. Yu Z, Gunn L, Brennan E, Reid R, Wall PG, Gaora PÓ, Hurley D, Bolton D, Fanning S: Complete genome sequence ofClostridium estertheticumDSM 8809 , a microbe identified in spoiled vacuum packed beef. Front Microbiol 2016, 7:1764-1764

60. Coil D, Jospin G, Darling AE: A5-miseq: an updated pipeline to assemble microbial genomes from Illumina MiSeq data. Bioinformatics 2014, 31(4):587-589.

61. Simão FA, Waterhouse RM, loannidis $P$, Kriventseva EV, Zdobnov EM: BUSCO: assessing genome assembly and annotation completeness with single-copy orthologs. Bioinformatics 2015, 31(19):3210-3212.

62. Meier-Kolthoff JP, Göker M: TYGS is an automated high-throughput platform for state-of-the-art genome-based taxonomy. Nat Commun 2019, 10(1):2182.

63. Lagesen $\mathrm{K}$, Hallin P, Rødland EA, Stærfeldt $\mathrm{H}-\mathrm{H}$, Rognes $\mathrm{T}$, Ussery DW: RNAmmer: consistent and rapid annotation of ribosomal RNA genes. Nucleic Acids Research 2007, 35(9):3100-3108.

64. Meier-Kolthoff JP, Auch AF, Klenk H-P, Göker M: Genome sequence-based species delimitation with confidence intervals and improved distance functions. BMC Bioinform 2013, 14(1):60.

65. Lefort V, Desper R, Gascuel O: FastME 2.0: A Comprehensive, Accurate, and Fast Distance-Based Phylogeny Inference Program. Mol Biol Evol 2015, 32(10):2798-2800.

66. Kumar S, Stecher G, Li M, Knyaz C, Tamura K: MEGA X: Molecular evolutionary genetics analysis across computing platforms. Mol Biol Evol 2018, 35(6):1547-1549.

67. Altermann E, Lu J, McCulloch A: GAMOLA2, a comprehensive software package for the annotation and curation of draft and complete microbial genomes. Front Microbiol 2017, 8:346-346.

68. Cimermancic P, Medema Marnix H, Claesen J, Kurita K, Wieland Brown Laura C, Mavrommatis K, Pati A, Godfrey Paul A, Koehrsen M, Clardy J et al: Insights into secondary metabolism from a global analysis of prokaryotic biosynthetic gene clusters. Cell 2014, 158(2):412-421.

\section{Publisher's Note}

Springer Nature remains neutral with regard to jurisdictional claims in published maps and institutional affiliations.

Ready to submit your research? Choose BMC and benefit from:

- fast, convenient online submission

- thorough peer review by experienced researchers in your field

- rapid publication on acceptance

- support for research data, including large and complex data types

- gold Open Access which fosters wider collaboration and increased citations

- maximum visibility for your research: over $100 \mathrm{M}$ website views per year

At BMC, research is always in progress.

Learn more biomedcentral.com/submissions 